\title{
TOPOLOGICAL PROPERTIES OF FOUR-LAYERED NEURAL NETWORKS
}

\author{
M. Javaid ${ }^{1}$, M. Abbas ${ }^{2}$, Jia-Bao Liu ${ }^{3}$, W. C. Teh ${ }^{4}$, Jinde $\mathrm{Cao}^{5}$ \\ ${ }^{1}$ Department of Mathematics, School of Science, University \\ of Management and Technology, Lahore, Pakistan \\ ${ }^{2}$ Department of Mathematics, GC University, \\ Lahore 54000, Pakistan \\ ${ }^{3}$ School of Mathematics and Physics, Anhui Jianzhu University, \\ Hefei, P.R. China \\ ${ }^{4}$ School of Mathematical Sciences, \\ Universiti Sains Malaysia, 11800 USM \\ ${ }^{5}$ School of Mathematics, Southeast University, Nanjing, \\ Jiangsu, 210096, P.R. China \\ E-mail: \{javaidmath, abbas.mujahid, jdcaoseu\}@gmail.com, \\ liujiabaoad@163.com,dasmenteh@usm.my \\ Submitted: 15th July 2018; Accepted: 14th October 2018
}

\begin{abstract}
A topological property or index of a network is a numeric number which characterises the whole structure of the underlying network. It is used to predict the certain changes in the bio, chemical and physical activities of the networks. The 4-layered probabilistic neural networks are more general than the 3-layered probabilistic neural networks. Javaid and Cao [Neural Comput. and Applic., DOI 10.1007/s00521-017-2972-1] and Liu et al. [Journal of Artificial Intelligence and Soft Computing Research, 8(2018), 225-266] studied the certain degree and distance based topological indices (TI's) of the 3-layered probabilistic neural networks. In this paper, we extend this study to the 4-layered probabilistic neural networks and compute the certain degree-based TI's. In the end, a comparison between all the computed indices is included and it is also proved that the TI's of the 4-layered probabilistic neural networks are better being strictly greater than the 3-layered probabilistic neural networks.
\end{abstract}

Keywords: degree of node, topological properties, neural network, probabilistic neural network

\section{Introduction}

A network which is obtained from nerve tissue and nervous system as a computer system modelled is called neural network. The probabilistic neural networks $\left(P N N^{S}\right)$ are studied to solve a num- ber of problems in the different areas of engineering, medical, chemistry, computer and mathematics, see [39]. In particular, for the enhancement of the email security systems and the intrusion detection systems [43, 44], to verify the signature [2], to identify damage localization for bridges and the 
effectiveness for ships [33, 35], to predict the stability of breakwaters [29], for detecting resistivity to antibiotics and diagnosing hepatitis [7, 5], for the segmentation and quantification of brain tissues from the certain type of images [48], and for the characterization of genetic variations in metabolic responses [25]. These networks are also used in the environmental sciences, see [41]. Moreover, to know about the different properties of the $P N N^{s}$, we refer [31].

More recently, the certain topological indices of the 3-layered $P N N^{s}$ are computed for the significant useful in the chemical industry, particularly in pharmaceutical and biotechnology research, see $[23,24,27,28]$. In the present note, we extend this study to the 4-layered $P N N^{s}$ and compute the certain degree-based TI's in the continuation of the progressive application role of the $P N N^{s}$ in the physical sciences.

A topological index (TI) is a numerical number which is used to predict various physical, biological and chemical activities such as surface tension, density, melting point, a heat of evaporation, and theboiling point of the involved chemical compound [8, 18, 30, 34, 37]. Moreover, it studies quantitative structure-activity relationships (QSAR) and structure-property relationshiprs (QSPR) that are used in the subject of cheminformatics. For further studies, we refer $[10,11,21]$.

In the subject of chemistry, first of all Wiener (1947) used a distance-based TI to compute the boiling point of paraffin [46]. But, degree-based TI's are most studied indices, see the recent survey [19]. TI's of the various networks such as nanostar dendrimers, silicate, honeycomb, hexagonal, carbon nanotube, alkane, and hydrocarbons networks are studied in [14, 15, 38, 12, 20, 4].

This paper includes the results related to the TI's which base on the degrees of the nodes and their neighbors for the 4-layered $P N N^{s}$. The rest of the current work is settled as: the frequent used definitions and formulas are given in Section 2, the main results of the TI's for the 4-layered $P N N^{s}$ are computed in Section 3 and a comparison between all the obtained indices is given in Section 4. In the same Section, we also show that the TI's of the 4-layered $P N N^{s}$ are better than the TI's of the 3layered $P N N^{s}$.

\section{Mathematical Preliminaries}

For the vertex-set $V(\Gamma)$ and the edge-set $E(\Gamma)$, a molecular graph $\Gamma=(V(\Gamma), E(\Gamma))$ is a graph in which vertices show atoms of the underlying chemical structure and edges present bonds between them. The number of vertices lying on a vertex $s$ is called its degree $(d(s))$. Now, we define $S(s)=\sum_{r \in N_{\Gamma}(s)}[d(r)]$ and $S(r)=\sum_{s \in N_{\Gamma}(r)}[d(s)]$, where $N_{\Gamma}(s)=\{r \in V(\Gamma) \mid s r \in E(\Gamma)\}$ and $N_{\Gamma}(r)=\{s \in$ $V(\Gamma) \mid s r \in E(\Gamma)\}$. If an edge connects a vertex to itself is called a loop and two or more than two edges are multiple edges if their end points are same. The used notions and terminologies of the graphs are standard, see [26, 47]. Now, we define some frequently used TI's.

Definition 2.1. The first Zagreb index, second Zagreb index, first general Zagrab index, generalized Zagreb index, first multiplicative Zagreb index, second multiplicative Zagreb index and general Randić index of the graph $\Gamma$ are defined

$$
\begin{aligned}
& M_{1}(\Gamma)=\sum_{r s \in E(\Gamma)}[d(r)+d(s)] \\
& M_{2}(\Gamma)=\sum_{r s \in E(\Gamma)}[d(r) \times d(s)] \\
& M_{1}^{\alpha}=\sum_{r \in V(\Gamma)}[d(r)]^{\alpha}=\sum_{r s \in E(\Gamma)}[d(r)+d(s)]^{\alpha} \\
& M_{p, q}(\Gamma)=\sum_{r s \in E(\Gamma)}\left[d(r)^{p} d(s)^{q}+d(r)^{q} d(s)^{p}\right] \\
& P M_{1}(\Gamma)=\prod_{r s \in E(\Gamma)}[d(r)+d(s)] \\
& P M_{2}(\Gamma)=\prod_{r s \in E(\Gamma)}[d(r) \times d(s)] \text { and } \\
& R_{\alpha}(\Gamma)=\sum_{r s \in E(\Gamma)}[d(r) \times d(s)]^{\alpha} \text { respectively. }
\end{aligned}
$$

In the general Randić index, for $\alpha=-\frac{1}{2}, \frac{1}{2}$ and 1 , we obtain Randić, reciprocal Randić and second Zagreb index respectively. For the detailed studies, we refer (Gutman and Trinajsti; 1972) [20], (Milan Randić; 1975) [36], Bollobás and Erdös; 1998) [6], (Amic et al.; 1998) [1], (Li and Zheng; 2005) [50], (Li and Gutman; 2006) [49], (Iranmanesh and Azari; 2011) [3], (Ghorbani and Azimi; 2012) [16]. 
Definition 2.2. The atom bond connectivity index, forth version of atom bond connectivity index, geometric-arithmetic index, fifth version of the geometric-arithmetic index, augmented Zagreb index and Sanskruti index are defined of the molecular graph $\Gamma$ as

$$
\begin{aligned}
& A B C(\Gamma)=\sum_{r s \in E(\Gamma)}\left[\sqrt{\frac{d(r)+d(s)-2}{d(r) \times d(s)}}\right], \\
& A B C_{4}(\Gamma)=\sum_{r s \in E(\Gamma)}\left[\sqrt{\frac{S(r)+S(s)-2}{S(r) \times S(s)}}\right], \\
& G A(\Gamma)=\sum_{r s \in E(\Gamma)}\left[\frac{2 \sqrt{d(r) \times d(s)}}{d(r)+d(s)}\right], \\
& G A_{5}(\Gamma)=\sum_{r s \in E(\Gamma)}\left[\frac{2 \sqrt{S(r) \times S(s)}}{S(r)+S(s)}\right], \\
& A Z I(\Gamma)=\sum_{r s \in E(\Gamma)}\left[\frac{d(r) \times d(s)}{d(r)+d(s)-2}\right]^{3} \text { and } \\
& S(\Gamma)=\sum_{r s \in E(\Gamma)}\left[\frac{S(r) \times S(s)}{S(r)+S(s)-2}\right]^{3} \text { respectively. }
\end{aligned}
$$

For the further studies of the above TI's, we refre (Estrada et al.; 1998)[12], (Ghorbani and Hosseinzadeh; 2010) [15], (Vukičević and Furtula; 2009) [45], (Graovac et al.; 2011) [14]. (Furtula et al.; 2010) [13] and (Sunilkumar M. Hosamani; 2016) [42].

The 4-layered $P N N^{s}$ consist of four layers of neurons (nodes) such as (i) input layer with a certain number of nodes (assume $r$ ), (ii) pattern layer with certain number of classes (say $s$ ) such that each class has $t$ nodes, (iii) summation layer with an equal number of nodes as of the pattern layer and (iv) output layer consists of exactly one node. In the architecture of the 4-layered $P N N^{s}$, each vertex of input layer is connected to all the vertices of each class of the pattern layer, all the vertices of each class of the pattern layer are connected to a corresponding single vrtex of the summation layer and all the nodes of the summation layer are connected with the output node. Thus order and size of the 4layered probabilistic neural network $P N N(r, s, t, 1)$ are $|V(P N N(r, s, t, 1))|=v=r+s(t+1)+1$ and $|E(P N N(r, s, t, 1))|=e=r s t+s t+s$ respectively, where $r, s$ and $t$ are natural numbers. In Figure 1, the 4-layered $P N N$ is shown.

\section{Main Results}

In this Section, 4-layered $P N N^{s}$ are discussed and the certain degree-based topological indices are computed. Before the main results, we explain our solution method as follows:

We make the vertex and edge partitions of the $P N N^{s}$ on the bases of the degrees of the vertices. We also formulate the cardinalities of all the obtained sets as the results of the partitions. Then, we apply the formulas and obtain the mathematical expressions in the most suitable forms. At the end, a comparison is also made with the help of the diagrams. There are four types of vertices in $P N N(r, s, t, 1)$, namely of degrees $s t, r+1, t+1$, and $s$. Thus, we have

$$
\begin{aligned}
& V_{1}=\{u \in V(\operatorname{PNN}(r, s, t, 1)) \mid d(u)=s t\}, \\
& V_{2}=\{u \in V(\operatorname{PNN}(r, s, t, 1)) \mid d(u)=1+r\}, \\
& V_{3}=\{u \in V(\operatorname{PNN}(r, s, t, 1)) \mid d(u)=1+t\}, \\
& V_{4}=\{u \in V(\operatorname{PNN}(r, s, t, 1)) \mid d(u)=s\},
\end{aligned}
$$

where $\left|V_{1}\right|=r,\left|V_{2}\right|=s t,\left|V_{3}\right|=s$ and $\left|V_{4}\right|=1$. Consequently, $|V(P N N(r, s, t, 1))|=v=\left|V_{1}\right|+\left|V_{2}\right|+$ $\left|V_{3}\right|+\left|V_{4}\right|=r+s(t+1)+1$. There are three types of edges in $P N N(r, s, t, 1)$ on the base of degrees of end vertices, namely $\{s t, r+1\},\{r+1, t+1\}$ and $\{t+1, s\}$. Thus, we have

$$
\begin{aligned}
& E_{1}=E_{\{s t, r+1\}} \\
& =\{u v \in E(P N N(r, s, t, 1)) \mid d(u)=s t, d(v)=r+1\}, \\
& E_{2}=E_{\{r+1, t+1\}} \\
& =\{u v \in E(P N N(r, s, t, 1)) \mid d(u)=r+1, d(v)=t+1\}, \\
& E_{3}=E_{\{t+1, s\}} \\
& =\{u v \in E(P N N(r, s, t, 1)) \mid d(u)=t+1, d(v)=s\},
\end{aligned}
$$

where $\quad\left|E_{\{s t, r+1\}}\right|=r s t, \quad\left|E_{\{r+1, t+1\}}\right|=s t$ and $\left|E_{\{t+1, s+1\}}\right|=s . \quad$ Consequently, $|E(P N N(r, s, t, 1))|=e=\left|E_{1}\right|+\left|E_{2}\right|+\left|E_{3}\right|=$ $r s t+s t+s$. Now, we define the vertex and edge partitions of $P N N(r, s, t, 1)$ on the base of degree sum of the neighbors of end vertices for each edge. Thus, see the following table for the partition sets of $E(P N N(r, s, t, 1))$. 


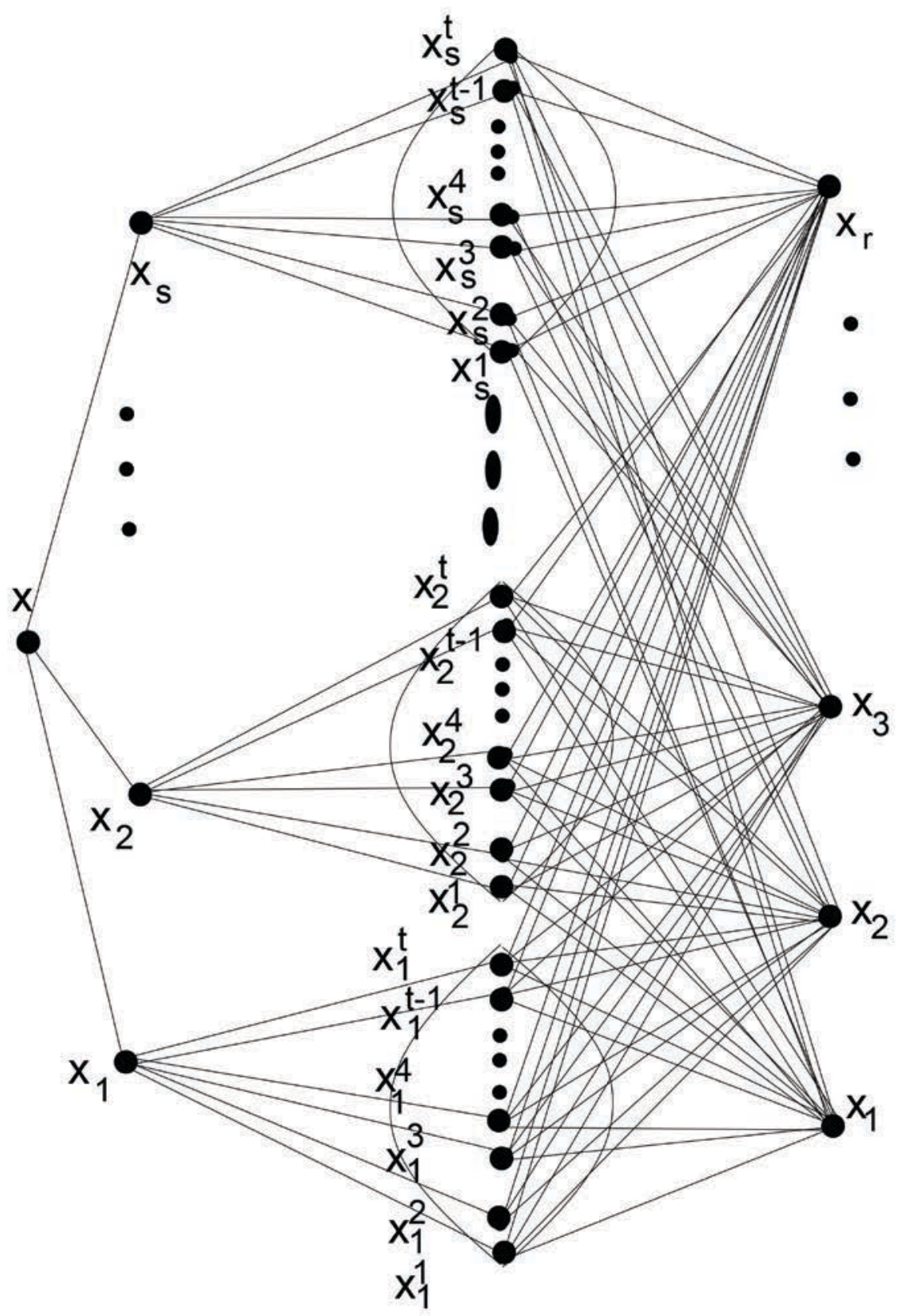

Figure 1. The 4-layered probabilistic neural network $P N N(r, s, t, 1)$. 
Table 1. Degree based edge partitions of $P N N(r, s, t, 1)$

\begin{tabular}{|l|l|l|l|}
\hline$E_{\{d(u), d(v)\}}$ & $E_{\{s t, r+1\}}$ & $E_{\{r+1, t+1\}}$ & $E_{\{t+1, s\}}$ \\
\hline$\left|E_{\{d(u), d(v)\}}\right|$ & $r s t$ & $s t$ & $s$ \\
\hline
\end{tabular}

Table 2. Degree sum of the neighbors based edge partitions of $P N N(r, s, t, 1)$

\begin{tabular}{|l|l|}
\hline$E_{\{S(u), S(v)\}}$ & $\left|E_{\{S(u), S(v)\}}\right|$ \\
\hline$E_{\{s t(r+1), r s t+t+1\}}$ & $r s t$ \\
\hline$E_{\{r s t+t+1, t(r+1)+s\}}$ & $s t$ \\
\hline$E_{\{t(r+1)+s, s(t+1)\}}$ & $s$ \\
\hline
\end{tabular}

Theorem 3.1. Let $\Gamma \cong P N N(r, s, t, 1)$ be a 4layered PNN. Then, for $r, s, t \geq 1$, its Randić index $\left(R_{-\frac{1}{2}}(\Gamma)\right)$, reciprocal Randić index $\left(R_{\frac{1}{2}}(\Gamma)\right)$, second Zagreb index $\left(R_{1}(\Gamma)\right)$ and the general Randic index $R_{\alpha}(\Gamma)$ are given as

$$
\begin{aligned}
& R_{\alpha}(\Gamma)= \\
& \left\{\begin{array}{l}
\sqrt{\frac{s}{(r+1)(t+1)}}[\sqrt{t}(r \sqrt{t+1}+\sqrt{s t})+\sqrt{r+1}], \\
\text { for } \alpha=-\frac{1}{2} \\
s[t \sqrt{r+1}(r \sqrt{s t}+\sqrt{t+1})+\sqrt{s(t+1)}], \\
\text { for } \alpha=\frac{1}{2} \\
s[t(r+1)(r s t+t+1)+s(t+1)], \\
\text { for } \alpha=1, \\
s\left[t(r+1)^{\alpha}\left\{r(s t)^{\alpha}+(t+1)^{\alpha}\right\}+\{s(t+1)\}^{\alpha}\right], \\
\text { for } \alpha \in \mathbb{R} .
\end{array}\right.
\end{aligned}
$$

Proof. For the required results, we use definitions of Section 2 and Tables of Section 3 as follow.

$$
\begin{aligned}
& R_{\alpha}(\Gamma)=\sum_{p q \in E(\Gamma)}[d(p) \times d(q)]^{\alpha} \\
& =\sum_{\left.p q \in E_{\{} s t, r+1\right\}}[d(p) \times d(q)]^{\alpha} \\
& +\sum_{\left.p q \in E_{\{} r+1, t+1\right\}}[d(p) \times d(q)]^{\alpha} \\
& +\sum_{\left.p q \in E_{\{} t+1, s\right\}}[d(p) \times d(q)]^{\alpha} \\
& =(r s t)[s t(r+1)]^{\alpha}+(s t)[(r+1)(t+1)]^{\alpha}+s[s(t+1)]^{\alpha}
\end{aligned}
$$

$=s\left[t(r+1)^{\alpha}\left\{r(s t)^{\alpha}+(t+1)^{\alpha}\right\}+\{s(t+1)\}^{\alpha}\right]$.

If we use $\alpha=-\frac{1}{2}, \frac{1}{2}$ and 1 , we obtain $R_{-\frac{1}{2}}(\Gamma)$, $R_{\frac{1}{2}}(\Gamma)$ ), and $R_{1}(\Gamma)$, respectively.

Theorem 3.2. Let $\Gamma \cong P N N(r, s, t, 1)$ be a 4-layered PNN. Then, for $r, s, t \geq 1, M_{1}^{\alpha}(\Gamma), M_{p, q}(\Gamma), P M_{1}(\Gamma)$ and $P M_{2}(\Gamma)$ indices are given by

(i) $M_{1}^{\alpha}(\Gamma)=s\left[t\left\{r(s t+r+1)^{\alpha}+(r+t+2)^{\alpha}\right\}+(s+\right.$ $\left.t+1)^{\alpha}\right]$,

(ii) $M_{p, q}(\Gamma)=s\left[(s)^{p}\left\{r t(t)^{p}(r+1)^{q}+(t+1)^{q}\right\}+\right.$ $t(r+1)^{p}\left\{r(s t)^{q}+(t+1)^{q}\right\}+(t+1)^{p}\left\{t(r+1)^{q}+\right.$ $\left.\left.s^{q}\right\}\right]$,

(iii) $P M_{1}(\Gamma)=\left[\left\{(s t+r+1)^{r}(t+r+2)\right\}^{t}(t+s+\right.$ 1) $]^{s}$,

(iv) $P M_{2}(\Gamma)=\left[\left\{(s t)^{r}(r+1)^{r+1}(t+1)\right\}^{t}\{s(t+1)\}\right]^{s}$.

Proof. For the required results, we use definitions of Section 2 and Tables of Section 3 as follow.

(i)

$$
\begin{aligned}
& M_{1}^{\alpha}(\Gamma)=\sum_{p q \in E(\Gamma)}[d(p)+d(q)]^{\alpha} \\
& =\sum_{\left.p q \in E_{\{} s t, r+1\right\}}[d(p)+d(q)]^{\alpha} \\
& +\sum_{\left.p q \in E_{\{r} r+1, t+1\right\}}[d(p)+d(q)]^{\alpha} \\
& +\sum_{\left.p q \in E_{\{} t+1, s\right\}}[d(p)+d(q)]^{\alpha} \\
& =(r s t)[s t+(r+1)]^{\alpha}+(s t)[(r+1)+(t+1)]^{\alpha} \\
& +s[s+(t+1)]^{\alpha} \\
& =s\left[t\left\{r(s t+r+1)^{\alpha}+(r+t+2)^{\alpha}\right\}+(s+t+1)^{\alpha}\right],
\end{aligned}
$$

(ii)

$$
\begin{aligned}
& M_{p, q}(\Gamma)=\sum_{u v \in E(\Gamma)}\left[d(u)^{p} d(v)^{q}+d(u)^{q} d(v)^{p}\right] \\
& =\sum_{\left.u v \in E_{\{s t}, r+1\right\}}\left[d(u)^{p} d(v)^{q}+d(u)^{q} d(v)^{p}\right] \\
& +\sum_{\left.u v \in E_{\{} r+1, t+1\right\}}\left[d(u)^{p} d(v)^{q}+d(u)^{q} d(v)^{p}\right] \\
& +\sum_{\left.u v \in E_{\{} t+1, s\right\}}\left[d(u)^{p} d(v)^{q}+d(u)^{q} d(v)^{p}\right] \\
& =(r s t)\left[(s t)^{p}(r+1)^{q}+(s t)^{q}(r+1)^{p}\right] \\
& +(s t)\left[(r+1)^{p}(t+1)^{q}+(r+1)^{q}(t+1)^{p}\right.
\end{aligned}
$$




$$
\begin{aligned}
& +\left[s^{p}(t+1)^{q}+s^{q}(t+1)^{p}\right] \\
& =s\left[(s)^{p}\left\{r t(t)^{p}(r+1)^{q}+(t+1)^{q}\right\}\right. \\
& +t(r+1)^{p}\left\{r(s t)^{q}+(t+1)^{q}\right\} \\
& \left.+(t+1)^{p}\left\{t(r+1)^{q}+s^{q}\right\}\right],
\end{aligned}
$$

(iii)

$$
\begin{aligned}
& P M_{1}(\Gamma)=\prod_{p q \in E(T)}[d(p)+d(q)] \\
& =\prod_{p q \in E_{\{s t, r+1\}}}[d(p)+d(q)] \times \prod_{p q \in E_{\{r+1, t+1\}}}[d(p)+d(q)] \\
& \times \prod_{p q \in E_{\{t+1, s\}}}[d(p)+d(q)] \\
& =[(s t)+(r+1)]^{r s t} \times[(r+1)+(t+1)]^{s t} \times[(t+1)+s]^{s} \\
& =\left[\left\{(s t+r+1)^{r}(t+r+2)\right\}^{t}(t+s+1)\right]^{s},
\end{aligned}
$$

$$
\begin{aligned}
& P M_{2}(\Gamma)=\prod_{p q \in E(T)}[d(p) \times d(q)] \\
& =\prod_{p q \in E_{\{s, r+1\}}}[d(p) \times d(q)] \times \prod_{p q \in E_{\{r+1, t+1\}}}[d(p) \times d(q)] \\
& \times \prod_{p q \in E_{\{t+1, s\}}}[d(p) \times d(q)] \\
& =[(s t)(r+1)]^{r s t} \times[(r+1)(t+1)]^{s t} \times[(t+1) s]^{s} \\
& =\left[\left\{(s t)^{r}(r+1)^{r+1}(t+1)\right\}^{t}\{s(t+1)\}\right]^{s} .
\end{aligned}
$$

The following corollary can be obtain with the help of Theorem 3.2.

Corollary to Theorem 3.2. Let $\Gamma \cong P N N(r, s, t, 1)$ be a 4-layered PNN. Then, for $r, s, t \geq 1, F(\Gamma)$ and $H M(\Gamma)$ are given by

$$
\begin{aligned}
& (i) F(\Gamma)=\sum_{p q \in E(\Gamma)}\left[(d(p))^{2}+(d(q))^{2}\right] \\
& =s\left[t(r+1)^{3}+(t+1)^{3}+s^{2}\left(r t^{3}+1\right)\right], \\
& (i i) H M(\Gamma)=\sum_{p q \in E(\Gamma)}[d(p)+d(q)]^{2} \\
& =s\left[t\left\{r(s t+r+1)^{2}+(r+t+2)^{2}\right\}+(s+t+1)^{2}\right] .
\end{aligned}
$$

Theorem 3.3. Let $\Gamma \cong P N N(r, s, t, 1)$ be a 4-layered PNN. Then, for $r, s, t \geq 1, A B C(\Gamma), G A(\Gamma) A Z I$ are given by

(i) $A B C(\Gamma)=\sqrt{\frac{s}{t(t+1)(r+1)}}[t\{r \sqrt{(t+1)(s t+r-1)}$ $+\sqrt{s t(r+t)}\}+\sqrt{t(r+1)(s+t-1)}]$,

(ii) $G A(\Gamma)=(2 s)\left[t \sqrt{r+1}\left\{r \frac{\sqrt{s t}}{s t+r+1}+\frac{\sqrt{t+1}}{r+t+2}\right\}+\right.$ $\left.\left(\frac{\sqrt{s(t+1)}}{s+t+1}\right)\right]$,

(iii) $A Z I(\Gamma)=s\left[t(r+1)^{3}\left\{r\left(\frac{s t}{s t+r-1}\right)^{3}+\left(\frac{t+1}{r+t}\right)^{3}\right\}+\right.$ $\left.\left(\frac{s(t+1)}{t+s-1}\right)^{3}\right]$.

Proof. For the required results, we use definitions of Section 2 and Tables of Section 3 as follow. (i)

$$
\begin{aligned}
& A B C(\Gamma)=\sum_{p q \in E(\Gamma)} \sqrt{\frac{d(p)+d(q)-2}{d(p) \times d(q)}} \\
& =\sum_{\left.p q \in E_{\{} s t, r+1\right\}}\left[\sqrt{\left.\frac{d(p)+d(q)-2}{d(p) \times d(q)}\right]}\right. \\
& +\sum_{\left.p q \in E_{\{} r+1, t+1\right\}}\left[\sqrt{\frac{d(p)+d(q)-2}{d(p) \times d(q)}}\right] \\
& +\sum_{\left.p q \in E_{\{} t+1, s\right\}}\left[\sqrt{\left.\frac{d(p)+d(q)-2}{d(p) \times d(q)}\right]}\right. \\
& =(r s t) \sqrt{\frac{(s t)+(r+1)-2}{(s t) \times(r+1)}} \\
& +(s t) \sqrt{\frac{(r+1)+(t+1)-2}{(r+1) \times(t+1)}} \\
& +(s) \sqrt{\frac{(t+1)+(s)-2}{(s) \times(t+1)}} \\
& \left.=\sqrt{\frac{s}{t(t+1)(r+1)}[t\{r \sqrt{(t+1)(s t+r-1)}}+\sqrt{s t(r+t)}\right\} \\
& +\sqrt{t(r+1)(s+t-1)}],
\end{aligned}
$$

$$
\begin{aligned}
& G A(\Gamma)=\sum_{p q \in E(\Gamma)} \frac{2 \sqrt{d(p) \times d(q)}}{d(p)+d(q)} \\
& =\sum_{\left.p q \in E_{\{} s t, r+1\right\}}\left[\frac{2 \sqrt{d(p) \times d(q)}}{d(p)+d(q)}\right] \\
& +\sum_{\left.p q \in E_{\{} r+1, t+1\right\}}\left[\frac{2 \sqrt{d(p) \times d(q)}}{d(p)+d(q)}\right]
\end{aligned}
$$




$$
\begin{aligned}
& +\sum_{\left.p q \in E_{\{} t+1, s\right\}}\left[\frac{2 \sqrt{d(p) \times d(q)}}{d(p)+d(q)}\right] \\
& =(r s t)\left[\frac{2 \sqrt{s t \times(r+1)}}{s t+(r+1)}\right]+(s t)\left[\frac{2 \sqrt{(r+1) \times(t+1)}}{(r+1)+(t+1)}\right] \\
& +s\left[\frac{2 \sqrt{s \times(t+1)}}{s+(t+1)}\right] \\
& =(2 s)\left[t\left\{r \frac{\sqrt{s t \times(r+1)}}{s t+(r+1)}+\frac{\sqrt{(r+1) \times(t+1)}}{(r+1)+(t+1)}\right\}\right. \\
& \left.+\left(\frac{\sqrt{s \times(t+1)}}{s+(t+1)}\right)\right] \\
& =(2 s)\left[t \sqrt{r+1}\left\{r \frac{\sqrt{s t}}{s t+r+1}+\frac{\sqrt{t+1}}{r+t+2}\right\}\right. \\
& \left.+\left(\frac{\sqrt{s(t+1)}}{s+t+1}\right)\right],
\end{aligned}
$$$$
\text { (iii) }
$$$$
\begin{aligned}
& A Z I(T)=\sum_{p q \in E(T)}\left[\frac{d(p) \times d(q)}{d(p)+d(q)-2}\right]^{3} \\
& =\sum_{\left.p q \in E_{\{} s t, r+1\right\}}\left[\frac{d(p) \times d(q)}{d(p)+d(q)-2}\right]^{3} \\
& +\sum_{\left.p q \in E_{\{} r+1, t+1\right\}}\left[\frac{d(p) \times d(q)}{d(p)+d(q)-2}\right]^{3} \\
& +\sum_{\left.p q \in E_{\{} t+1, s\right\}}\left[\frac{d(p) \times d(q)}{d(p)+d(q)-2}\right]^{3} \\
& \quad=(r s t)\left[\frac{(s t) \times(r+1)}{(s t)+(r+1)-2}\right]^{3} \\
& \quad+(s t)\left[\frac{(r+1) \times(t+1)}{(r+1)+(t+1)-2}\right]^{3} \\
& +s\left[\frac{s \times(t+1)}{t+s-1}\right]^{3} \\
& \quad=s\left[t(r+1)^{3}\left\{r\left(\frac{s t}{s t+r-1}\right)^{3}+\left(\frac{t+1}{r+t}\right)^{3}\right\}\right. \\
& \left.+\left(\frac{s(t+1)}{t+s-1}\right)^{3}\right] .
\end{aligned}
$$

Theorem 3.4. Let $\Gamma \cong P N N(r, s, t, 1)$ be a 4-layered PNN. Then, for $r, s, t \geq 1, A B C_{4}, G A_{5}$, and $S$ are given as (i) $\quad A B C_{4}(\Gamma)=s\left[\frac{t}{\sqrt{r s t+t+1}}\left\{r \sqrt{\frac{2 r s t+s t+t-1}{s t(r+1)}}+\right.\right.$ $\left.\left.\sqrt{\frac{r s t+r t+2 t+s-1}{t r+t+s}}\right\}+\sqrt{\frac{r t+s t+t+2 s-2}{s(r t+t+s)(t+1)}}\right]$,

(ii) $G A_{5}(\Gamma)=2 s\left[t \sqrt{r s t+t+1}\left\{r\left(\frac{\sqrt{s t(r+1)}}{2 r s t+s t+t+1}\right)+\right.\right.$ $\left.\left.\frac{\sqrt{t r+t+s}}{r s t+t r+2 t+s+1}\right\}+\frac{\sqrt{s(t r+t+s)(t+1)}}{t r+s t+2 s+t}\right]$,

(iii) $S(\Gamma)=s\left[t(r s t+t+1)^{3}\left\{r\left(\frac{s t(r+1)}{2 r s t+s t+t-1}\right)^{3}+\right.\right.$ $\left.\left.\left(\frac{(t r+t+s)}{r s t+t r+2 t+s-1}\right)^{3}\right\}+\left(\frac{s(t r+t+s)(t+1)}{t r+s t+2 s+t-2}\right)^{3}\right]$.

Proof. For the required results, we use definitions of Section 2 and Tables of Section 3 as follow.

$$
\begin{aligned}
& A B C_{4}(\Gamma)=\sum_{p q \in E(\Gamma)} \sqrt{\frac{S(p)+S(q)-2}{S(p) \times S(q)}} \\
& =\sum_{\left.p q \in E_{\{} s t(r+1), r s t+t+1\right\}} \sqrt{\frac{S(p)+S(q)-2}{S(p) \times S(q)}}
\end{aligned}
$$$$
+\sum_{\left.p q \in E_{\{} r s t+t+1, t(r+1)+s\right\}} \sqrt{\frac{S(p)+S(q)-2}{S(p) \times S(q)}}
$$$$
+\sum_{\left.p q \in E_{\{t} t(r+1)+s, s(t+1)\right\}} \sqrt{\frac{S(p)+S(q)-2}{S(p) \times S(q)}}
$$$$
=(r s t) \sqrt{\frac{s t(r+1)+(r s t+t+1)-2}{s t(r+1) \times(r s t+t+1)}}
$$$$
+(s t) \sqrt{\frac{(r s t+t+1)+(r t+t+s)-2}{(r s t+t+1) \times(t r+t+s)}}
$$$$
+(s) \sqrt{\frac{(r t+t+s)+s(t+1)-2}{(r t+t+s) \times(s t+s)}}
$$$$
=s\left[t \left\{r \sqrt{\frac{2 r s t+s t+t-1}{s t(r+1)(r s t+t+1)}}\right.\right.
$$$$
\left.+\sqrt{\frac{r s t+r t+2 t+s-1}{(r s t+t+1)(t r+t+s)}}\right\}
$$

$\left.+\sqrt{\frac{r t+s t+t+2 s-2}{s(r t+t+s)(t+1)}}\right]$

$=s\left[\frac{t}{\sqrt{r s t+t+1}}\left\{r \sqrt{\frac{2 r s t+s t+t-1}{s t(r+1)}}\right.\right.$

$\left.+\sqrt{\frac{r s t+r t+2 t+s-1}{t r+t+s}}\right\}$

$\left.+\sqrt{\frac{r t+s t+t+2 s-2}{s(r t+t+s)(t+1)}}\right]$,

(ii)

$G A_{5}(\Gamma)=\sum_{p q \in E(\Gamma)}\left[\frac{2 \sqrt{S(p) \times S(q)}}{S(p)+S(q)}\right]$ 


$$
\begin{aligned}
& =\sum_{\left.p q \in E_{\{} s t(r+1), r s t+t+1\right\}}\left[\frac{2 \sqrt{S(p) \times S(q)}}{S(p)+S(q)}\right] \\
& +\sum_{\left.p q \in E_{\{} r s t+t+1, t(r+1)+s\right\}}\left[\frac{2 \sqrt{S(p) \times S(q)}}{S(p)+S(q)}\right] \\
& +\sum_{\left.p q \in E_{\{t} t(r+1)+s, s(t+1)\right\}}\left[\frac{2 \sqrt{S(p) \times S(q)}}{S(p)+S(q)}\right] \\
& =(r s t)\left[\frac{2 \sqrt{s t(r+1) \times(r s t+t+1)}}{(r s t+s t)+(r s t+t+1)}\right] \\
& +(s t)\left[\frac{2 \sqrt{(r s t+t+1) \times(t r+t+s)}}{(r s t+t+1)+(t r+t+s)}\right] \\
& +(s)\left[\frac{2 \sqrt{(t r+t+s) \times(s t+s)}}{(t r+t+s)+(s t+s)}\right] \\
& =2 s\left[t \left\{r \frac{\sqrt{s t(r+1)(r s t+t+1)}}{2 r s t+s t+t+1}\right.\right. \\
& \left.+\frac{\sqrt{(r s t+t+1)(t r+t+s)}}{r s t+t r+2 t+s+1}\right\} \\
& \left.+\frac{\sqrt{s(t r+t+s)(t+1)}}{t r+s t+2 s+t}\right] \\
& =2 s\left[t \sqrt { r s t + t + 1 } \left\{r\left(\frac{\sqrt{s t(r+1)}}{2 r s t+s t+t+1}\right)\right.\right. \\
& \left.+\frac{\sqrt{t r+t+s}}{r s t+t r+2 t+s+1}\right\} \\
& \left.+\frac{\sqrt{s(t r+t+s)(t+1)}}{t r+s t+2 s+t}\right]
\end{aligned}
$$

$$
\begin{aligned}
& S(\Gamma)=\sum_{p q \in E(\Gamma)}\left[\frac{S(p) \times S(q)}{S(p)+S(q)-2}\right]^{3} \\
& =\sum_{\left.p q \in E_{\{} s t(r+1), r s t+t+1\right\}}\left[\frac{S(p) \times S(q)}{S(p)+S(q)-2}\right]^{3} \\
& +\sum_{\left.p q \in E_{\{r} r t+t+1, t(r+1)+s\right\}}\left[\frac{S(p) \times S(q)}{S(p)+S(q)-2}\right]^{3} \\
& +\quad \sum_{\left.p q \in E_{\{} t(r+1)+s, s(t+1)\right\}}\left[\frac{S(p) \times S(q)}{S(p)+S(q)-2}\right]^{3} \\
& =(r s t)\left[\frac{s t(r+1) \times(r s t+t+1)}{s t(r+1)+(r s t+t+1)-2}\right]^{3} \\
& +(s t)\left[\frac{(r s t+t+1) \times(t r+t+s)}{(r s t+t+1)+(t r+t+s)-2}\right]^{3}
\end{aligned}
$$

$$
+(s)\left[\frac{(t r+t+s) \times(s t+s)}{(t r+t+s)+(s t+s)-2}\right]^{3}
$$$$
\begin{aligned}
& =s\left[t ( r s t + t + 1 ) ^ { 3 } \left\{r\left(\frac{s t(r+1)}{2 r s t+s t+t-1}\right)^{3}\right.\right. \\
& \left.+\left(\frac{(t r+t+s)}{r s t+t r+2 t+s-1}\right)^{3}\right\}
\end{aligned}
$$$$
\left.+\left(\frac{s(t r+t+s)(t+1)}{t r+s t+2 s+t-2}\right)^{3}\right] .
$$

\section{Conclusion}

This Section includes the comparison between all the computed TI's for the 4-layered $P N N^{s}$. In Section 3, the results are obtained in term of $r$ (total vertices in first layer), $s$ (number of classes in pattern layer and number of nodes in summation layer) and $t$ (number of nodes in each class of pattern layer). Moreover, $|V(P N N(r, s, t, 1))|=v=$ $r+s(t+1)+1$. If we assume $s=2$ and $t=1$, then the 4-layered PNN becomes $P N N(r, 2,1,1)$ with or$\operatorname{der} v=r+5$.

In Figure 2, the values of $v$ and the computed TI's of the 4-layered PNN $(P N N(r, 2,1,1))$ are taken along the horizontal and vertical line respectively. It can be noted that the TI's $M_{1}, M_{2}, M_{1,1}$, $A Z I, A B C, A B C_{4}, G A$ and $G A_{5}$ remain constant such that $M_{2}$ and $M_{1,1}$ are dominant. However, the Sanskurti index $(\mathrm{S})$ is rapidly increasing with the increasing values of $v$. In Figure $3, P M_{1}, F$, and $H M$ are constant with $H M$ as a dominant index and $P M_{2}$ rapidly increases with the increasing values of $v$. Now in Figure 4, we find $P M_{2}$ as a dominant than the $S$ index which shows that $P M_{2}$ is better one among all the computed indices.

Now, to prove that the TI's of the 4-layered $P N N^{s}$ are better than the topological indices of the 3-layered $P N N^{s}$, we only show that $P M_{2}$ of the 4-layered $P N N^{s}$ is greater than the $P M_{2}$ of the 3layered $P N N^{s}$. For the purpose, we proceed as follows. 


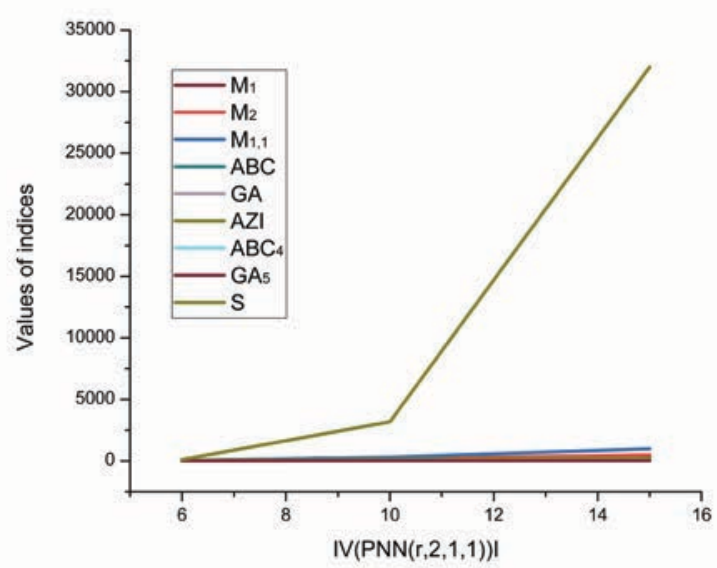

Figure 2. $S$ is shown as a better index than $M_{1}, M_{2}$, $M_{1,1}, A Z I, A B C, A B C_{4}, G A$ and $G A_{5}$ for the 4-layered probabilistic neural network

$$
P N N(r, 2,1,1) \text {. }
$$

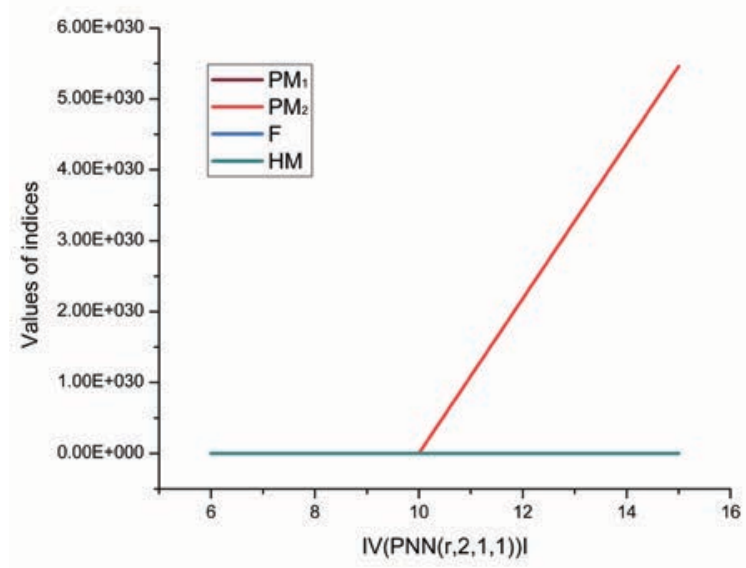

Figure 3. $P M_{2}$ is shown as a better index than $P M_{1}, F$ and $H M$ for the 4-layered probabilistic neural network $P N N(r, 2,1,1)$

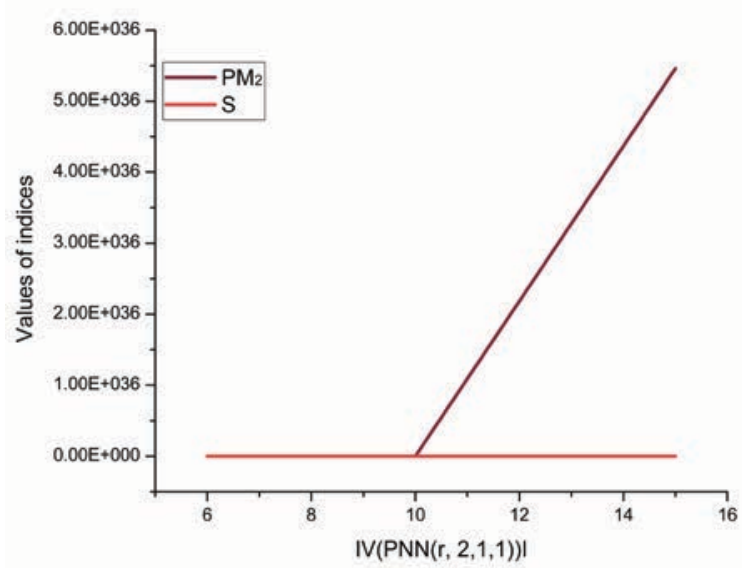

Figure 4. Comparison between the $S$ and $P M_{2}$ topological indices of $P N N(r, 2,1,1)$

Consider,

$(s)^{r t}(t)^{r t}(r+1)^{t(r+1)}=(s)^{r t}(t)^{r t}(r+1)^{t(r+1)}$

Since for positive integral values of $s$ and $t,(t+$ $1)^{t+1} s>(t)^{t}$, we have

$(s)^{r t}(t)^{r t}(r+1)^{t(r+1)}(t+1)^{t+1} s>$

$(s)^{r t}(t)^{r t}(r+1)^{t(r+1)}(t)^{t}$

$\left[(s)^{r t}(t)^{r t}(r+1)^{t(r+1)}(t+1)^{t+1} s\right]^{s}>$

$\left[(s)^{r t}(t)^{r t}(r+1)^{t(r+1)}(t)^{t}\right]^{s}$

$\left[\left\{(s t)^{r}(r+1)^{r+1}(t+1)\right\}^{t}\{s(t+1)\}\right]^{s}>$

$\left[(s)^{r t}(t(r+1))^{t(r+1)}\right]^{s}$,

where $\left[\left\{(s t)^{r}(r+1)^{r+1}(t+1)\right\}^{t}\{s(t+1)\}\right]^{s}$ is $P M_{2}$ of the 4-layered $P N N^{s}(P N N(r, s, t, 1))$ and $\left[(s)^{r t}(t(r+1))^{t(r+1)}\right]^{s}$ is $P M_{2}$ of the 3-layered $P N N^{s}(P N N(r, s, t))$. The last inequality shows that the $P M_{2}$ of the 4-layered $P N N^{s}(P N N(r, s, t, 1))$ is greater than the $P M_{2}$ of the 3-layered $P N N^{s}$ $(P N N(r, s, t))$. For further study of the 3-layered $P N N^{s}$, we refer [27]. Hence, we conclude that the obtained TI's can be helpful to understand the topological properties of the 4-layered $P N N^{s}$. For the uses of these indices in various fields particularly in pharmaceutical industry, see [24, 23, 27, 19]. 


\section{Acknowledgement}

The authors would like to express their sincere gratitude to the anonymous referees for their insightful comments and valuable suggestions, which led to a number of improvements in the earlier version of this manuscript.

\section{References}

[1] D. Amic, D. Beslo, B. Lucic, S. Nikolic, N. Trinajstić, The vertex-connectivity index revisited, J. Chem. Inf. Comput. Sci. 38 (1998) 819-822

[2] L.F. Araghi, H. Khaloozade, M.R. Arvan, Ship identification using probabilistic neural networks. In: Proceedings of the international multiconference of engineers and computer scientists, 2(2009), $18-20$

[3] M. Azari, A. Iranmanesh, Generalized Zagreb index of graphs, Studia Univ. Babes-Bolyai. 56 (3) (2011) 59-70

[4] M. Bača, J. Horváthová, M. Mokrišová, Andrea Semaničová-Feňovčková, and A. Suhńniov, On topological indices of a carbon nanotube network, Can. J. Chem. 93(2015), 1157-1160

[5] M.S. Bascil, H. Oztekin, A study on hepatitis disease diagnosis using probabilistic neural network, J. Med. Syst. 36(2012), 1603-1606

[6] B. Bollobs, P. Erdös, Graphs of extremal weights, Ars Combin. 50 (1998) 225-233

[7] F. Ü. Budak, E.D. beyli, Detection of resistivity for antibiotics by probabilistic neural networks, J. Med. Syst. 35(2011), 87-91

[8] F.M. Brückler, T. Došlíc, A. Graovac, I. Gutman, On a class of distance-based molecular structure descriptors, Chem. Phys. Lett. 503, (2011), 336338

[9] A.-S. Chen, M.T. Leung, H. Daouk, Application of neural networks to an emerging financial market: forecasting and trading the Taiwan Stock Index, Computers and Operations Research, 30, (2003), 901923

[10] J. Devillers, A.T. Balaban, Topological Indices and Related Descriptors in QSAR and QSPR, Gordon Breach, Amsterdam, 1999.

[11] M.V. Diudea (Ed.), QSPR/QSAR Studies by Molecular Descriptors, NOVA, New York, 2001.

[12] E. Estrada, L. Torres, L. Rodríguez, I. Gutman, An atom-bond connectivity index: modelling the enthalpy of formation of alkanes, Indian J. Chem. 37 A(1998) 849-855.
[13] B. Furtula, A. Graovac, D. Vukičević, Augmented Zagreb index, J. Math. Chem. 48(2010), 370-380.

[14] A. Graovac, M. Ghorbani, M.A. Hosseinzadeh, Computing fifth geometricarithmetic index for nanostar dendrimers, J. Math. Nanosci. 1 (1) (2011) 33-42.

[15] M. Ghorbani, M.A. Hosseinzadeh, Computing $A B C_{4}$ index of nanostar dendrimers, Optoelectron. Adv. Mater.-Rapid Commun. 4 (9) (2010) 14191422.

[16] M. Ghorbani, N. Azimi, Note on multiple Zagreb indices, Iran. J. Math. Chem. 3 (2) (2012) 137-143.

[17] W. Gao, W. Wang, and M.R. Farahani, Topological indices study of molecular structure in anticancer drugs, Journal of Chemistry, (2016), Doi:org/10.1155/2016/3216327.

[18] H. González-Díaz, S. Vilar, L. Santana and E. Uriarte, Medicinal Chemistry and Bioinformatics Current Trends in Drugs Discovery with Networks Topological Indices, Current Topics in Medicinal Chemistry, 7(10), (2007), 1015-1029.

[19] I. Gutman, Degree-based topological indices, Croat. Chem. Acta (2013), 86, 351-361.

[20] I. Gutman, N. Trinajsti, Graph theory and molecular orbitals. III. Total $\pi$-electron energy of alternant hydrocarbons, Chem. Phys. Lett. 17(1972) 535538.

[21] I. Gutman, O. Polansky, Mathematical Concepts in Organic Chemistry, Springer-Verlag, Berlin, 1986.

[22] I. Gutman Degree-based topological indices. Croat. Chem. Acta 86, (2013), 351-361.

[23] H. González-Díaz, S. Vilar, L. Santana and E. Uriarte, Medicinal Chemistry and Bioinformatics Current Trends in Drugs Discovery with Networks Topological Indices, Current Topics in Medicinal Chemistry, 2007, 7 (10), 1015-1029.

[24] L.H. Hall, L.B. Kier, Molecular Connectivity in Chemistry and Drug Research; Academic Press: Boston, 239 MA, USA, 1976.

[25] E. Holmes, J. K. Nicholson, G. Tranter, Metabonomic characterization of genetic variations in toxicological and metabolic responses using probabilistic neural networks, Chemical Research in Toxicology, 14(2)(2001), 182-191.

[26] F. Harary, Graph Theory, Addison-Wesley 1969.

[27] M. Javaid, Jinde Cao, Computing topological indices of probabilistic neural network, Neural Comput. and Applic., DOI 10.1007/s00521-017-29721. 
[28] J.-B. Liu, J. Zhao, S. Wang, M. Javaid, J. Cao, On the topological properties of the certain neural networks, Journal of Artificial Intelligence and Soft Computing Research, 8(2018), 225-266.

[29] D. Kim, D.H. Kim, S. Chang, Application of probabilistic neural network to design breakwater armor blocks, Ocean Engineering, 2008, 35, 294300 .

[30] S. Klavžar and I. Gutman, A Comparison of the Schultz molecular topological index with the Wiener index, J. Chem. Inf. Comput. Sci., 36, (1996), 1001-1003.

[31] P.A. Kowalski, P. Kulczycki, Interval probabilistic neural network, Neural Comput. Applic. DOI 10.1007/s00521-015-2109-3.

[32] J.K. Labanowski, I. Motoc and R.A. Dammkoehler, The physical meaning of topological indices, Computers Chem., 1(15), (1991), 47-53.

[33] J.-J. Lee, C.-B. Yun, Damage localization for bridges using probabilistic neural networks, KSCE Journal of Civil Engineering 11, 2(2007), 111-120.

[34] A. R. Matamala and E. Estrada, Generalised topological indices: Optimisation methodology and physico-chemical interpretation, Chemical Physics Letters, 410, (2005), 343-347.

[35] S. Meshoul, M. Batouche, A novel approach for online signature verification using fisher based probabilistic neural network, In: Proceedings of IEEE symposium on computers and communications, (2010), 314-319.

[36] M. Randić , On characterization of molecular branching, J. Am. Chem. Soc. 97 (1975) 66096615.

[37] G. Rucker, C. Rucker, On topological indices, boiling points, and cycloalkanes, J. Chem. Inf. Comput. Sci., 39, (1999), 788-802.

[38] B. Rajan, A. William, C. Grigorious and S. Stephen, On certain topological indices of silicate, honeycomb and hexagonal networks, J. Comp. Math. Sci. Vol.3 5(2012), 530-535.

[39] D.F. Specht, Probabilistic neural networks, Neural Netw 3(1990), 109-118.
[40] F. Shafiei, Relationship between topological indices and thermodynamic properties and of the monocarboxylic acids applications in QSPR, Iranian Journal of Mathematical Chemistry, 1(6), (2015), 15-28.

[41] I.B. Standal, J. Rainuzzo, D.E. Axelson, S. Valdersnes, K. Julshamn, M. Aursand, Classification of geographical origin by PNN analysis of fatty acid data and level of contaminants in oils from Peruvian anchovy. J Am Oil Chem Soc 89(7)(2012), 1173-1182.

[42] Sunilkumar M. Hosamani, Computing Sanskruti index of certain nanostructures, J. Appl. Math. Comput. DOI 10.1007/s12190-016-1016-9.

[43] T. Tran, T. Nguyen, P. Tsai, X. Kong, BSPNN: boosted subspace probabilistic neural network for email security. Artif Intell Rev 35(2011), 369-382.

[44] T.P. Tran, L. Cao, D. Tran, C.D Nguyen, Novel intrusion detection using probabilistic neural network and adaptive boosting. Int. J. Comput. Sci. Inf. Secur 6(2009)83-91.

[45] D. Vukičević , B. Furtula, Topological index based on the ratios of geometrical and arithmetical means of end-vertex degrees of edges, J. Math. Chem. 46(2009) 1369-1376.

[46] H.J. Wiener, Structural determination of paraffin boiling points, J. Amer. Chem. Soc. 69 (1947) 1720.

[47] D.B. West, Introduction to Graph Theory, USA Printce Hall 1996.

[48] Y. Wang, T. Adali, S. Y. Kung, Z. Szabo, Quantification and segmentation of brain tissues from MR images: a probabilistic neural network approach, Ieee Transactions on Image Processing, 7(8)(1998), 1165-1181.

[49] X. Li, I. Gutman, Mathematical aspects of Randictype molecular structure descriptors, Mathematical Chemistry Monographs, No.1, Kragujevac, 2006.

[50] X. Li and J. Zheng, A unified approach to the extremal trees for different indices, MATCH Commun. Math. Comput. Chem. 54 (2005), 195208.

[51] F. Yan, Q. Shang, S. Xia, Q. Wang, and P. Ma, Application of topological index in predicting ionic liquids densities by the quantitative structure property relationship method, J. Chem. Eng. Data, 60, (2015), 734-739. 


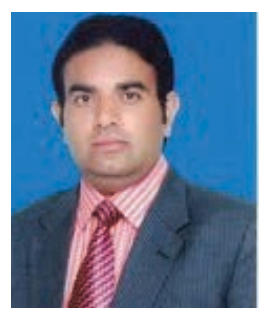

Muhammad Javaid received the M.Sc and M.Phil. degrees from University of the Punjab and GC University, Lahore Pakistan in 2002 and 2008 respectively. He completed his Ph.D Mathematics (2009-14) from National University of Computer and Emerging Sciences, Lahore, Pakistan and Post Doctorate Mathematics (2015-17) from School of Mathematical Sciences, University of Science and Technology of China, Hefei, China. He is currently working as an Assistant Professor of Mathematics at the Department of Mathematics, School of Science, University of Management and Technology, Lahore, Pakistan. His research interests are in different areas of graph theory such as spectral theory of graphs, computational graph theory and chemical graph theory.

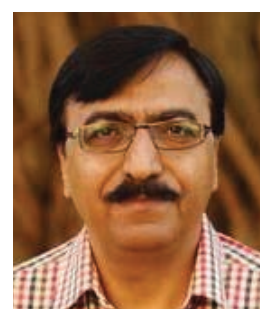

Mujahid Abbas, is a Professor of Mathematics in Department of Mathematics, Government College University Lahore, Pakistan, extraordinary Professor in Department of Mathematics and Applied Mathematics, University of Pretoria, South Africa and Distinguished Professor (Adjunct): Department of Mathematics, King Abdulaziz University Saudi Arabia. He is National Research Foundation (South Africa) rated mathematician. He is also Thomson Reuters highly cited researcher 2015, 2016 and 2017. He is an associate editor of The Scientific World Journal, Creative Math. Inform. Sci., the Journal of Advanced Topology, Journal of Mathematical Analysis and has served as guest editor for Journal of Function Spaces. Currently, he is the guest editor for Journal of Function Spaces and Guest Editor, Special Issue 2017 Recent Developments in Generalized Metric Spaces and Fixed Point Results with Applications, Journal of Mathematics.

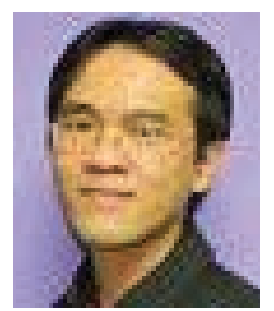

W. C. Teh, is currently working as a Senior Lecturer at School of Mathematical Sciences, Universiti Sains Malaysia, Malaysia. He received the B.S. degree in Pure Mathematics and M.S. degree in Mathematics in 2001 and 2004 respectively from Universiti Sains Malaysia, Malaysia. He completed his PhD Mathematics in 2012 from the Ohio State University, USA. He is working in different areas of research in Mathematics such as Combinatorics, Ramsey Theory and Theory of Natural Computing.

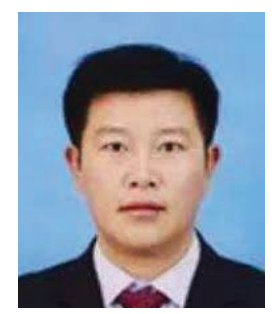

Jia-Bao Liu is currently an Associate Professor at School of Mathematics and Physics, Anhui Jianzhu University, Hefei 230601, China. He received the B.S. degree in Mathematics and Applied Mathematics from Wanxi University, Anhui, China, in 2005, and the M.S. degree and Ph.D. degree in Mathematics and Applied Mathematics from Anhui University, Anhui, China, in 2009 and 2016, respectively. From September 2013 to July 2014, he was a Visiting Researcher at the School of Mathematics, Southeast University, China. In March 2017, he was a Postdoctoral Fellow at the School of Mathematics, Southeast University, China. His current research interests include graph theory and its applications, fractional calculus theory, neural networks, and complex dynamical networks. Dr. Liu is a Reviewer of Mathematical Reviews and Zentralblatt-Math.

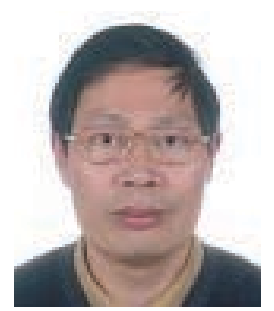

Jinde Cao is a Distinguished Professor, the Dean of School of Mathematics and the Director of the Research Center for Complex Systems and Network Sciences at Southeast University. He is Associate Editor of the Journal of the Franklin Institute, Neurocomputing, the IEEE Transactions on Cybernetics, Differential Equations and Dynamical. Systems, Mathematics, and Computers in Simulation, and Neural Networks. He is a Fellow of IEEE, a Member of the Academy of Europe, a Fellow of Pakistan Academy of Sciences, and a member of European Academy of Sciences and Arts. He is Highly-Cited Researcher in three fields named by Thomson Reuters/Clarivate Analytics. 